\title{
Expanding the Use of a Fluorogenic Method to Determine Activity and Mode of Action of Bacillus thuringiensis Bacteriocins Against Gram-Positive and Gram-Negative Bacteria
}

\author{
Norma M. de la Fuente-Salcido, ${ }^{1,2}$ J. Eleazar Barboza-Corona, ${ }^{2}$ \\ A. N. Espino Monzón, ${ }^{1}$ R. D. Pacheco Cano, ${ }^{2}$ N. Balagurusamy, ${ }^{1}$ \\ Dennis K. Bideshi, ${ }^{3,4}$ and Rubén Salcedo-Hernández ${ }^{2}$ \\ ${ }^{1}$ Escuela de Ciencias Biológicas, Universidad Autónoma de Coahuila, 27440 Torreón, COAH, Mexico \\ ${ }^{2}$ División Ciencias de la Vida, Departamento de Alimentos, Universidad de Guanajuato Campus Irapuato-Salamanca, \\ 36500 Irapuato, GTO, Mexico \\ ${ }^{3}$ Department of Natural and Mathematical Sciences, California Baptist University, 8432 Magnolia Avenue, Riverside, \\ CA 92504, USA \\ ${ }^{4}$ Department of Entomology, University of California, Riverside, CA 92521, USA
}

Correspondence should be addressed to Norma M. de la Fuente-Salcido, normapbr322@hotmail.com

Received 16 April 2012; Accepted 8 May 2012

Academic Editors: S. Fuchs, K. Hong, E. Parrilli, and L. Ramirez

Copyright () 2012 Norma M. de la Fuente-Salcido et al. This is an open access article distributed under the Creative Commons Attribution License, which permits unrestricted use, distribution, and reproduction in any medium, provided the original work is properly cited.

\begin{abstract}
Previously we described a rapid fluorogenic method to measure the activity of five bacteriocins produced by Mexican strains of Bacillus thuringiensis against $B$. cereus 183 . Here we standardize this method to efficiently determine the activity of bacteriocins against both Gram-positive and Gram-negative bacteria. It was determined that the crucial parameter required to obtain reproducible results was the number of cells used in the assay, that is, $\sim 4 \times 10^{8} \mathrm{cell} / \mathrm{mL}$ and $\sim 7 \times 10^{8}$ cell $/ \mathrm{mL}$, respectively, for target Gram-positive and Gram-negative bacteria. Comparative analyses of the fluorogenic and traditional well-diffusion assays showed correlation coefficients of 0.88 to 0.99 and 0.83 to 0.99 , respectively, for Gram-positive and Gram-negative bacteria. The fluorogenic method demonstrated that the five bacteriocins of $B$. thuringiensis have bacteriolytic and bacteriostatic activities against all microorganisms tested, including clinically significant bacteria such as Listeria monocytogenes, Proteus vulgaris, and Shigella flexneri reported previously to be resistant to the antimicrobials as determined using the well-diffusion protocol. These results demonstrate that the fluorogenic assay is a more sensitive, reliable, and rapid method when compared with the well-diffusion method and can easily be adapted in screening protocols for bacteriocin production by other microorganisms.
\end{abstract}

\section{Introduction}

Antimicrobial peptides are biomolecules synthesized by both eukaryotic and prokaryotic organisms. They are involved in multiple functions, such as protection against the attack by microorganisms, endotoxin neutralization, chemotactic and immunomodulating activities, induction of angiogenesis, and wound repair [1]. Antimicrobial peptides produced by bacteria are called bacteriocins [2]. Among the most studied bacteriocins are those synthesized by Lactobacillus species. However, the screening of new microorganisms that produce antimicrobial peptides with biotechnological applied use has been expanded to other types of bacteria, including Bacillus spp. In this regard, Bacillus thuringiensis, one of the most important bioinsecticide used worldwide, synthesizes different types of bacteriocins that elaborate inhibitory activity against a rich diversity of bacteria including human pathogens, and those involved in food spoilage, foulbrood disease of honeybees, and crop diseases [3-11]. In particular, Morricin 269, Kurstacin 287, Kenyacin 404, Entomocin 420, and Tolworthcin 524 are bacteriocins produced by Mexican strains of $B$. thuringiensis that are active in a wide range 
of temperatures and $\mathrm{pH}$ against Gram-positive and Gramnegative bacteria, and thus could be of applied interest to industry [10].

As the search for viable and stable bacteriocins for use in the food and medicinal industries is expanding, developing new rapid and sensitive protocols could facilitate screening and identification of microbes that produce these peptides. Methods for detecting bacteriocin producers are based on enzymatic and nonenzymatic procedures, the former being a more sensitive technique [12-14]. Recently, an innovative and rapid fluorogenic method for detecting bacteriocin activity based on berberine fluorescence following its influx into compromised cells of Bacillus cereus 183 was described [15]. Using this fluorogenic method, bacteriocin activity was accurately determined within one hour which was much more rapid than conventional methods that are tedious and time consuming, especially methods employing welldiffusion assays $[13,14]$.

In order to expand the applied use of the fluorogenic method, here we standardize the conditions to evaluate the antimicrobial activity of the five bacteriocins synthesized by B. thuringiensis against both Gram-positive and Gram-negative pathogenic bacteria and also determine the underlying modes of action of these antimicrobial peptides. The method is sensitive, rapid, and reliable and can be easily adapted to screen and identify bacteriocin activities of other microbes. In addition, we show that the bacteriocins produced by $B$. thuringiensis elicit both bacteriolytic and bacteriostatic effects against target microorganisms of clinical significance.

\section{Material and Methods}

2.1. Bacterial Strains. Bacteriocin-producing strains, used in this study, were Bacillus thuringiensis subsp. morrisoni (LBIT 269), B. thuringiensis subsp. kurstaki (LBIT 287), B. thuringiensis subsp kenyae (LBIT 404), B. thuringiensis subsp. entomocidus (LBIT 420), and B. thuringiensis subsp. tolworthi (LBIT 524) obtained from a native bacterial stock collection held at CINVESTAV, Campus Guanajuato, Mexico. These strains synthesize Morricin 269, Kurstacin 287, Kenyacin 404, Entomocin 420, and Tolworthcin 524, respectively [11]. Bacillus cereus 183 was obtained from a collection of Bacillus strains maintained in the International Entomopathogenic Bacillus Centre, Institute Pasteur in Paris, France and was used as the indicator bacterium for the determination of bacteriocin activity in well diffusion method. Activity of the bacteriocins were determined against Gram-positive (Staphylococcus xylosus ATCC 700404, S. aureus ATCC 25923, Bacillus cereus 183, B. subtilis ATCC 6633, Listeria monocytogenes, Micrococcus species ATCC 700405, Streptococcus agalactiae, Streptococcus pyogenes, and Enterococcus faecalis ATCC 10541) and Gram-negative bacteria (Pseudomonas aeruginosa ATCC 27853, Proteus vulgaris ATCC 13315, Escherichia coli ATCC 25932, Klebsiella pneumoniae, Enterobacter cloacae ATCC 13047, Salmonella sp., Salmonella typhimurium ATCC 14022, Serratia marcescens Nima, Serratia marcescens WF, Shigella flexneri, and Shigella sonnei).
2.2. Bacteriocin Production. Each producer strain was cultivated in tryptic soy broth (TSB) for the time where the highest bacteriocin activity was detected in kinetic studies as previously described [10]. Cultures were centrifuged at $10,000 \times \mathrm{g}$ for $15 \mathrm{~min}$, and the supernatant was filtered through $0.20 \mathrm{~mm}$ filter. Supernatants were concentrated with ammonium sulfate to $80 \%$ saturation and precipitated at $4^{\circ} \mathrm{C}$ with constant stirring overnight. Precipitated proteins were pelleted by centrifugation at $16,000 \times \mathrm{g}$ for $30 \mathrm{~min}$ at $4^{\circ} \mathrm{C}$, resuspended in $100 \mathrm{mM}$ phosphate buffer ( $\mathrm{pH} 7.0$ ), dialyzed overnight against the same buffer using a mini-dialysis kit with a $1 \mathrm{kDa}$ cutoff (Amersham Biosciences), and stored at $-20^{\circ} \mathrm{C}$ for subsequent studies.

2.3. Adjustment of Bacterial Concentration. Depending on the strain tested, bacteria were grown overnight in TSB, Brain Heart Infusion (BHI), Luria-Bertani Broth (LB), Tetrathionate Broth (TB), Nutrient Broth (NB), de Man, Rogosa and Sharp Broth (MRSB), at 28 or $37^{\circ} \mathrm{C}$ and 200 $\mathrm{rpm}$ (see Table 2). One volume of each culture was mixed with 4 volumes of TSB and incubated at 28 or $37^{\circ} \mathrm{C}$, for $2 \mathrm{~h}$ at $200 \mathrm{rpm}$. Then cell pellets were obtained by centrifugation at $10,000 \times \mathrm{g}$ for $15 \mathrm{~min}$ and resuspended in $50 \mathrm{mM}$ phosphate buffer with $5 \%(\mathrm{v} / \mathrm{v})$ glycerol $(\mathrm{PBG})$ to adjust the bacterial concentration to $\sim 4 \times 10^{8}$ cell $/ \mathrm{mL}$ and $\sim 7 \times 10^{8}$ cell $/ \mathrm{mL}$, respectively, for Gram-positive and Gramnegative bacteria [15].

2.4. Evaluation of Antibacterial Activity by Fluorogenic Method. $20 \mu \mathrm{L}$ with $\sim 4 \times 10^{8}$ cell $/ \mathrm{mL}$ or $\sim 7 \times 10^{8} \mathrm{cell} / \mathrm{mL}$ were mixed with different volumes $(10,20,30,40,50 \mu \mathrm{L})$ of each bacteriocin, $24 \mu \mathrm{M}$ of berberine sulfate (Sigma), and $50 \mathrm{mM}$ PBG to reach a volume of $1000 \mu \mathrm{L}$. All samples were incubated at room temperature and fluorescence was determined in a Turner fluorometer (model 450; 340-nm interference filter and $415 \mathrm{~nm}$ cut filter). Triplicate fluorescence assays were performed and average values were plotted against bacteriocin concentrations. In addition, bacteria tested with each bacteriocin and berberine were observed by fluorescence microscopy with a ND filter (Eclipse E200, Nikon). Bacteria treated only with bacteriocins were stained with $0.005 \%(\mathrm{w} / \mathrm{v})$ amido black 10B (BioRad) in 50\% (v/v) ethanol and observed under light microscopy (Axio Imager A1, Carl Zeiss).

\subsection{Evaluation of Antibacterial Activity by Well-Diffusion} Assay. Concomitant with the fluorogenic assays, $25 \mathrm{~mL}$ of TSB with soft agar $0.7 \%(\mathrm{wt} / \mathrm{vol})$ was mixed with $50 \mu \mathrm{L}(\sim 1$ $\left.\times 10^{9} \mathrm{cell} / \mathrm{mL}\right)$ of each tested culture and plated. The same volumes $(10,20,30,40,50 \mu \mathrm{L})$ of bacteriocins used in the fluorescence were added to wells, $7 \mathrm{~mm}$ in diameter, and plates were incubated for $12 \mathrm{~h}$ at $4^{\circ} \mathrm{C}$ to allow the diffusion of the samples, followed by an additional incubation at 28 or $37^{\circ} \mathrm{C}$ for one day before diameters of zones of inhibition were measured. One unit (U) of activity was defined as $1 \mathrm{~mm}^{2}$ of the zone of inhibition of growth of the indicator bacterium [10]. Finally, fluorescence average values were plotted against average of activity determined by well-diffusion method and 
the correlation between both methodologies was established $[16,17]$.

2.6. Effect of Bacteriocins on the Growth of Bacterial Cultures. B. cereus and Salmonella sp. were selected to study the effect of bacteriocin on bacterial cultures. Both microorganisms were grown overnight and $\sim 1 \times 10^{9}$ cells $/ \mathrm{mL}$ was inoculated in $100 \mathrm{~mL}$ of fresh broth (TSB). B. cereus and Salmonella $s p$. cultures were incubated for $\sim 4$ and $3 \mathrm{hr}$ at $28^{\circ} \mathrm{C}$ or $37^{\circ} \mathrm{C}$, respectively, with constant stirring at $180 \mathrm{rpm}$ in an orbital shaking incubator (Shel Lab, Cornelius, OR, USA), to reach the middle of their logarithmic-phase (log phase), and then $\sim 3000 \mathrm{U}$ of bacteriocin was added to the flask. Units (U) were determined with the well-diffusion method using B. cereus as indicator bacterium as shown previously [10]. The optical density $\left(\mathrm{OD}_{660 \mathrm{~nm}}\right)$ was monitored at 5, 15, 45, and 60 minutes in triplicate assays using a Smartspec 3000 Spectrophotometer (Bio-Rad). Additionally, the number of viable cells ( $\mathrm{CFU} \mathrm{mL}^{-1}$ ) was determined by total viable count using samples serially diluted with saline solution $(0.85 \% \mathrm{w} / \mathrm{v})$ and plated onto plate count agar during $24 \mathrm{~h}$ at 28 or $37^{\circ} \mathrm{C}[6,18]$. In both cases, duplicate cultures were grown without adding bacteriocins and used as controls.

\section{Results}

3.1. Standardization of the Fluorogenic Method with a Gram-Negative Bacterium (Salmonella sp.). The fluorogenic method using berberine was previously standardized with a Gram-positive bacterium (i.e., B. cereus) [15]. In order to expand the practical application of this method to evaluate the bacteriocin activity to Gram-negative bacteria, we tested the susceptibility of Salmonella sp. using the fluorogenic method. We determined that conditions were similar to that reported in the $B$. cereus assay [17]. However, the crucial difference to obtain reproducible results was the number of cells used in the assay. For assays with Salmonella sp., it was necessary to use $\sim 7 \times 10^{8} \mathrm{cell} / \mathrm{mL}$ (optical density of $\sim 1.5$ ), compared to assays with $B$. cereus, where $\sim 4 \times 10^{8}$ cell $/ \mathrm{mL}$ (optical density of 0.5 to 1.0 ) [15] were required to obtain optimal and reproducible results.

In previous work [12] it was observed that bacteriocins tested against $B$. cereus 183 showed a linear increment of fluorescence with bacteriocin concentration, that is, the higher bacteriocin concentration, the higher fluorescence. Similar behavior was observed with Salmonella sp., using up to $50 \mu \mathrm{L}$ of each crude bacteriocin preparation. Correlation coefficients $(r)$ of each bacteriocin assayed separately were 0.96 for Kenyacin 404, 0.97 for Morricin 269, and 0.99 for Kurstacin 287, Entomocin 420, and Tolworthcin 524 (Figure 1(a)). When bacteriocins were evaluated against Salmonella sp. and activity was measured as the area of inhibition $\left(\mathrm{mm}^{2}\right)$ and also as the fluorescence emitted by berberine, a correlation of 0.94 was observed, suggesting that the fluorogenic method could be efficiently substituted for the well-diffusion method against Gram-negative bacteria. Accordingly with the assay against Salmonella sp., the maximum inhibition area of $100 \mathrm{~mm}^{2}$ that correspond to a relative fluorescence of 100 still gave a linear response (Figure 1(b)).

3.2. Correlation between the Fluorogenic Method and the WellDiffusion Assay. In order to confirm that bacteriocin activity determined by fluorescence correlates with the standard agar diffusion methods not only with $B$. cereus 183 [15] and Salmonella sp. but also with other Gram-negative and Grampositive bacteria, comparable experiments were performed in triplicate using the same conditions. When bacteriocins were tested against Gram-positive bacteria, fluorogenic and well-diffusion methods showed correlation coefficients $(r)$ of 0.88 to 0.99 . Likewise, they showed correlations of 0.83 to 0.99 when bacteriocins were assayed against Gram-negative bacteria (Table 1).

3.3. Susceptibility of Bacteria to Bacteriocins Tested with the Fluorogenic Method. The results described above confirmed the utility of the fluorogenic method to evaluate the inhibitory effect of bacteriocins against bacteria in a short period of time. Thus, the method was used to determine bacteriocin susceptibilities against Gram-negative bacteria such as $P$. aeruginosa, $P$. vulgaris, E. coli, K. pneumoniae, E. cloacae, Salmonella sp., S. typhimurium, S. marcescens Nima, S. marcescens $w f$, Shigella flexneri, and Shigella sonnei and also of Gram-positive, S. xylosus, S. aureus, B. cereus 183, B. subtilis, L. monocytogenes, Micrococcus sp., Str. agalactiae, Str. pyogenes, and E. faecalis (Table 2).

3.4. Pore-Forming Activity. When cells of Salmonella sp. were treated with bacteriocins, the cellular membrane was damaged allowing the entrance of berberine (a nonpermeable alkaloid) that fluoresces inside the cells (Figure 1(c)). It was observed that the higher bacteriocin concentration the higher fluorescence was emitted, indicating an increment in the cellular damage (Figure 1(a)). Similar results were recorded when $B$. cereus 183 was treated with bacteriocinsberberine and observed under fluorescence microscopy (data not shown). Likewise, an increment in the bacteriocin concentrations generated an augmentation of the fluorescence emitted by B. cereus [15].

3.5. Mode of Action of Bacteriocins Synthesized by Bacillus thuringiensis. The five bacteriocins of $B$. thuringiensis were added in the middle of the logarithmic phase of the growth curve of a Gram-positive (B. cereus 183) and Gram-negative (Salmonella sp.) bacterium, and the effect on the optical density and the bacterial viability was evaluated.

When bacteriocins were added to $B$. cereus, a decrement of $\sim 5-12$-fold in the indicator strain viable cell number was observed after 5 min of exposure, when compared with cells not treated with the antimicrobial peptides. Similarly, a diminution in the optical density was detected at $5 \mathrm{~min}$ of incubation. The decrease in the colony forming unit (CFU) and the optical density were maintained after a longer period of time (i.e., $60 \mathrm{~min}$ ) (Figure 2). These results suggested that the five bacteriocins of $B$. thuringiensis exhibited a 


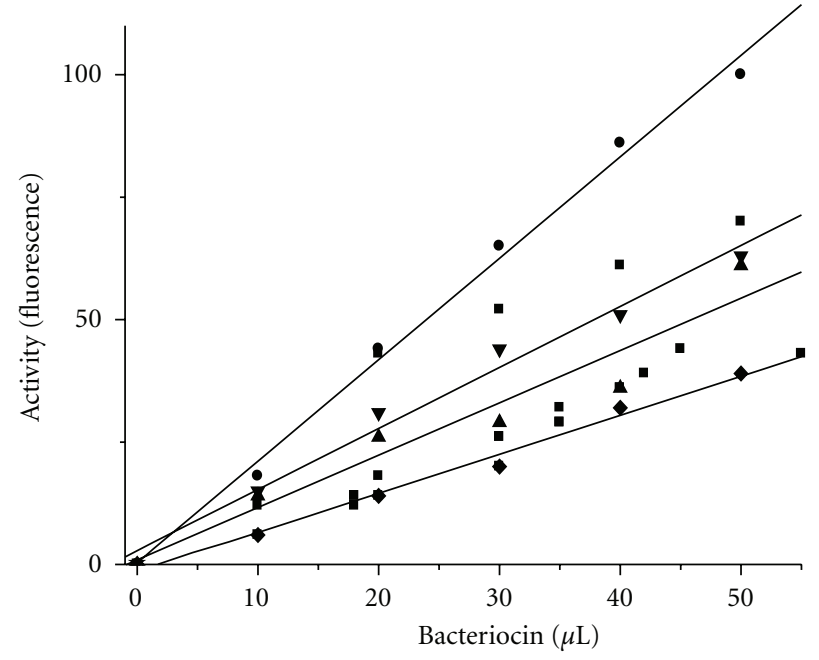

(a)

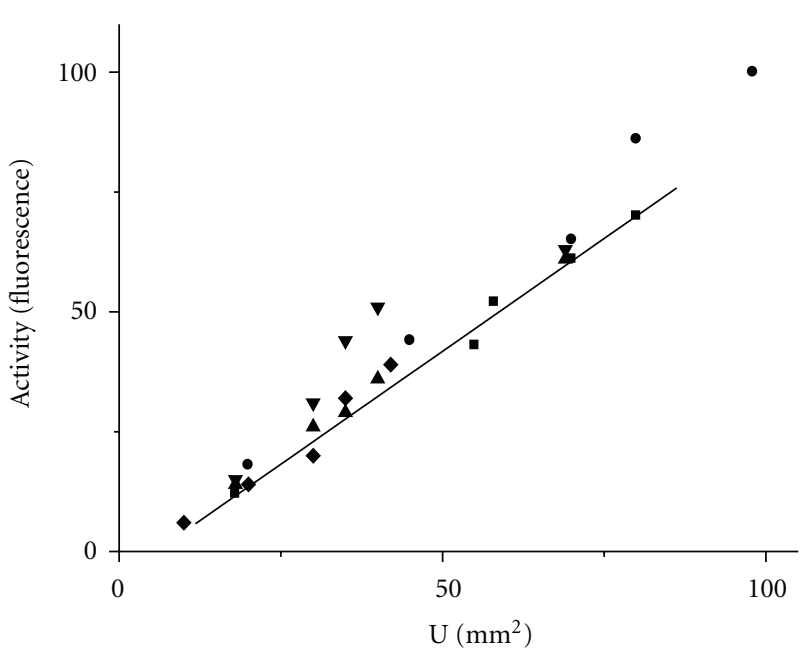

(b)

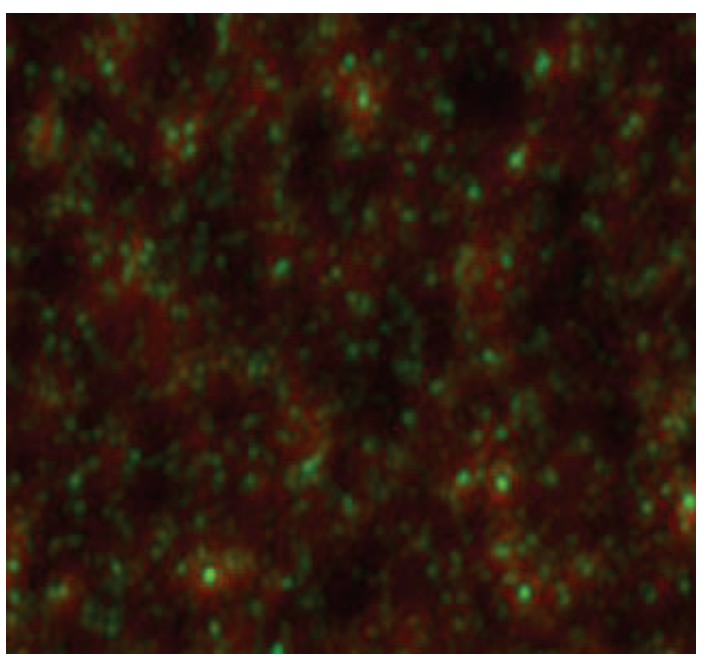

(c)

Figure 1: (a) Fluorescence emitted by berberine sulfate upon influx into Salmonella sp. treated with different concentrations of bacteriocins produced by B. thuringiensis. (b) Correlation $(r=0.94)$ between the bacteriocin activity in $\mathrm{mm}^{2}$ of inhibition area and fluorescence. Morricin $269(\boldsymbol{\nabla})$; Kurstacin $287(\bullet)$; Kenyacin $404(\boldsymbol{\Delta})$; Entomocin $420(\boldsymbol{\nabla})$; Tolworthcin $524(\boldsymbol{})$. (c) Fluorescence emitted by berberine sulfate inside Salmonella sp. after the effect on cytoplasmic membrane induced by bacteriocin Kurstacin 287 .

TABLE 1: Correlation coefficient between the fluorogenic method and well diffusion assay.

\begin{tabular}{|c|c|c|c|c|c|}
\hline \multirow{2}{*}{ Strain } & \multicolumn{5}{|c|}{ Correlation } \\
\hline & Morricin 269 & Kurstacin 287 & Kenyacin 404 & Entomocin 420 & Tolworthcin 524 \\
\hline \multicolumn{6}{|l|}{ Gram-negative } \\
\hline Proteus vulgaris & 0.96 & 0.95 & 0.89 & 0.95 & 0.95 \\
\hline Klebsiella pneumoniae & 0.98 & 0.99 & 0.88 & 0.99 & 0.91 \\
\hline Salmonella sp. & 0.97 & 0.99 & 0.96 & 0.99 & 0.99 \\
\hline Shigella flexneri & 0.95 & 0.95 & 0.93 & 0.89 & 0.95 \\
\hline Shigella sonnei & 0.95 & 0.99 & 0.99 & 0.93 & 0.93 \\
\hline \multicolumn{6}{|l|}{ Gram-positive } \\
\hline Bacillus cereus 183 & 0.99 & 0.99 & 0.99 & 0.99 & 0.97 \\
\hline Bacillus subtilis & 0.87 & 0.90 & 0.94 & 0.94 & 0.83 \\
\hline Listeria monocytogenes & 0.92 & 0.93 & 0.94 & 0.96 & 0.98 \\
\hline Enterococcus faecalis & 0.94 & 0.94 & 0.99 & 0.95 & 0.99 \\
\hline
\end{tabular}


TABLE 2: Inhibitory activity (UF) of bacteriocins synthesized by B. thuringiensis determined by fluorogenic method.

\begin{tabular}{|c|c|c|c|c|c|c|}
\hline \multirow{2}{*}{ Strain } & \multicolumn{6}{|c|}{ Bacteriocin activity } \\
\hline & Morricin 269 & Kurstacin 287 & Kenyacin 404 & Entomocin 420 & Tolworthcin 524 & Culture media* ${ }^{*}{ }^{\circ} \mathrm{C}^{\mathrm{a}}$ \\
\hline \multicolumn{7}{|l|}{ Gram-negative } \\
\hline Pseudomonas aeruginosa & 74 & 109 & 120 & 81 & 106 & TSB \\
\hline Proteus vulgaris & 110 & 78 & 67 & 93 & 52 & TSB/BHI \\
\hline Escherichia coli & 77 & 97 & 120 & 68 & 100 & TSB/LB \\
\hline Streptococcus pyogenes & 80 & 109 & 123 & 79 & 109 & TSB \\
\hline Klebsiella pneumoniae & 76 & 96 & 114 & 82 & 115 & TSB \\
\hline Enterobacter cloacae & 101 & 93 & 110 & 67 & 101 & TSB \\
\hline Salmonella sp. & 70 & 126 & 61 & 63 & 39 & $\mathrm{TSB} / \mathrm{TB}$ \\
\hline Salmonella typhimurium & 71 & 105 & 128 & 82 & 121 & TSB/TB \\
\hline Serratia marcescens Nima & 114 & 97 & 110 & 70 & 108 & TSB \\
\hline Serratia marcescens $w f$ & 95 & 93 & 110 & 75 & 100 & TSB \\
\hline Shigella flexneri & 80 & 110 & 131 & 118 & 110 & TSB/TB \\
\hline Shigella sonnei & 70 & 84 & 105 & 66 & 98 & $\mathrm{TSB} / \mathrm{TB}$ \\
\hline \multicolumn{7}{|l|}{ Gram-positive } \\
\hline Staphylococcus xylosus & 87 & 77 & 98 & 133 & 100 & NB \\
\hline Staphylococcus aureus & 78 & 90 & 118 & 76 & 95 & NB \\
\hline Bacillus cereus 183 & 125 & 89 & 64 & 96 & $82^{\mathrm{a}}$ & $\mathrm{TSB} / \mathrm{LB}^{\mathrm{a}}$ \\
\hline Bacillus subtilis & 72 & 106 & 129 & 83 & $110^{\mathrm{a}}$ & $\mathrm{TSB} / \mathrm{LB}^{\mathrm{a}}$ \\
\hline Listeria monocytogenes & 103 & 96 & 110 & 67 & 96 & NB \\
\hline Micrococcus sp. & 111 & 100 & 122 & 71 & $106^{\mathrm{a}}$ & $\mathrm{TSB} / \mathrm{NB}^{\mathrm{a}}$ \\
\hline Streptococcus agalactiae & 106 & 90 & 73 & 91 & 50 & $\mathrm{NB} / \mathrm{BHI}$ \\
\hline Enterococcus faecalis & 109 & 98 & 125 & 73 & 104 & MRSB \\
\hline
\end{tabular}

${ }^{*}$ Culture media-TSB: Trypticase Soy Broth; BHI: Brain Heart Infusion; LB: Luria-Bertani Broth; TB: Tetrathionate Broth; NB: Nutrient Broth; MRSB: de Man, Rogosa and Sharp Broth.

${ }^{a}$ All bacteria were incubated at $37^{\circ} \mathrm{C}$, except B. cereus 183, B. subtilis, and Micrococcus sp., that were cultivated at $28^{\circ} \mathrm{C}$.

bacteriolytic effect against a Gram-positive bacterium. Bacteriolytic effect was confirmed when $B$. cereus was treated with Kurstacin 287 and observed under light microscopy after 5 min of incubation. A drastic effect occurred, where cells lost membrane integrity and lysed (Figure 3). A similar effect was observed with the other bacteriocins of $B$. thuringiensis (data not shown).

Addition of the five bacteriocins to cultures of Salmonella sp. showed that this bacterium was less susceptible than $B$. cereus 183 to the antimicrobial peptides. After $5 \mathrm{~min}$ of the addition of bacteriocins, a decrement in both viable cells and optical density was observed. However, over longer periods of incubation, almost parallel increases in both viable cell count and optical density was observed, although values were consistently lower than those of the control cells not treated with bacteriocins (Figure 4). These results suggested that bacteriocins of $B$. thuringiensis produced a low bacteriostatic effect against a Gram-negative bacterium.

\section{Discussion}

We initially standardized the fluorogenic method to determine the antibacterial activity of bacteriocins of $B$. thuringiensis against B. cereus 183 and also evaluated the utility of the methodology to determine the susceptibility of other Gram-positive bacterium (i.e., Listeria innocua) to Nisin and Pediocin [12]. Using similar parameters in that study, we were unable to obtain reproducible results against Gram-negative bacteria. Our present study shows that in order to expand this technology to include screenings against Gram-negative bacteria a significant increase in the number of cells was required, using Salmonella sp. as the model target. Although we do not have a specific reason why this is so, this observation is possibly related to the difference in cellular envelope composition between Gram-positive and Gramnegative bacteria $[19,20]$. Gram-negative bacteria have a lipopolysaccharide coat surrounding the peptidoglycan layer that could prolong penetration of berberine into the bacteriocin-treated cell. Thus reproducible collective fluorescence signals could be obtained with more cells present in the assay as compared to lower levels when fewer cells are used. It should be noted that similar increment in fluorescence was obtained with the other Gram-negative bacteria in assays with higher cell density.

Although bacteriocins vary in their spectrum of activity, mode of action, molecular weight, genetic origin, and biochemical properties [21], bacteriocins synthesized by a Gram-positive bacterium typically have activity only against other Gram-positive species[21-24]. We previously 


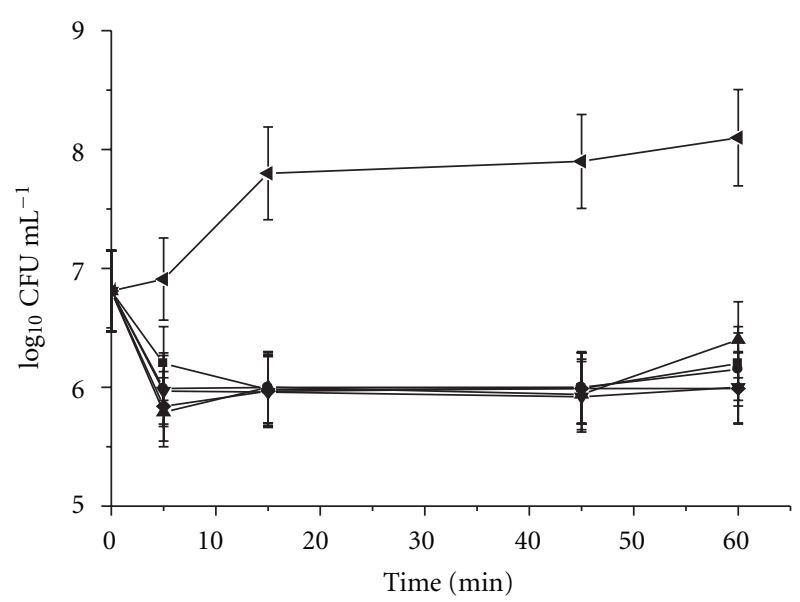

(a)

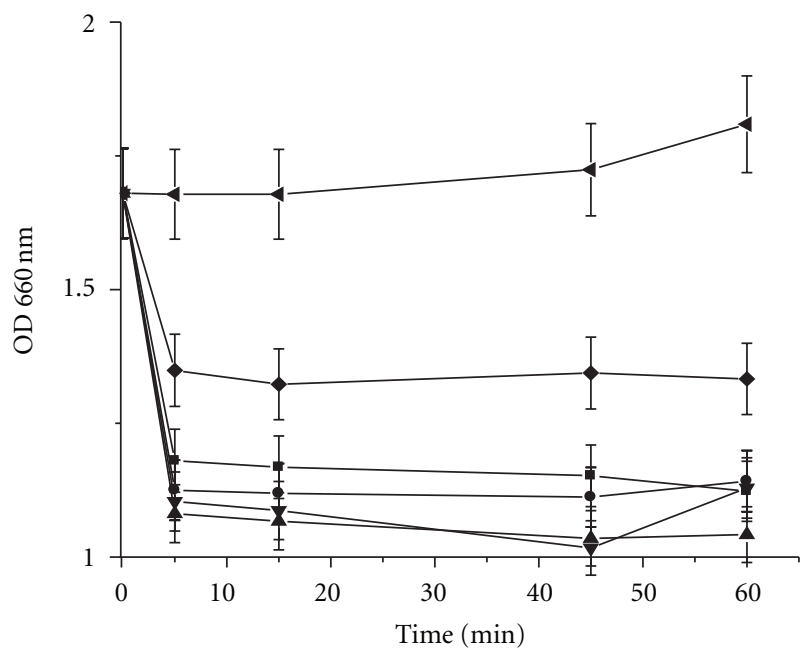

(b)

FIGURE 2: Effect of bacteriocins produced by B. thuringiensis on the growth of $B$. cereus 183 . (a) $\log _{10}$ cfu $\mathrm{mL}^{-1}$. (b) Optical density measured at $660 \mathrm{~nm}$. The five bacteriocins were added in the middle of the logarithmic phase of growth curve $(\sim 4 \mathrm{~h})$ of $B$. cereus (indicator strain) used as control (without bacteriocins). B. cereus $183(\varangle)$; Morricin $269(\boldsymbol{\square})$; Kurstacin $287(\bullet)$; Kenyacin $404(\boldsymbol{\Delta})$, Entomocin $420(\boldsymbol{\nabla})$; Tolworthcin $524(\diamond)$.

determined by the well-diffusion method that bacteriocins produced by Mexican strains of $B$. thuringiensis have inhibitory effect against both Gram-positive and Gramnegative bacteria [25-29], which was confirmed in this work by the fluorogenic method. In this regard, antibacterial activity of bacteriocins as determined by the fluorogenic and the well-diffusion methods against Gram-positive and Gram-negative bacteria showed a high level of correlation coefficients, indicating that the flourogenic method can substitute accurately for the well-diffusion method. In addition, because of the reliability of the fluorogenic methodology, we were able to detect the susceptibility of a different class of bacteria to bacteriocins of $B$. thuringiensis not observed by the well-diffusion method. For example, we reported that the

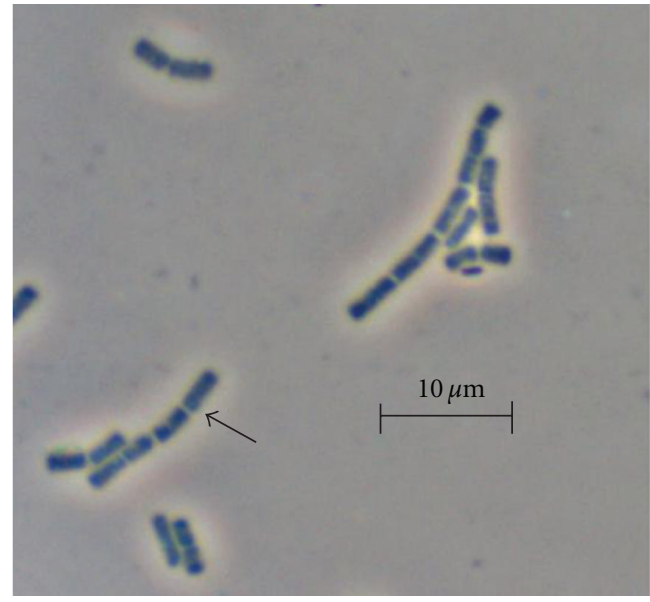

(a)

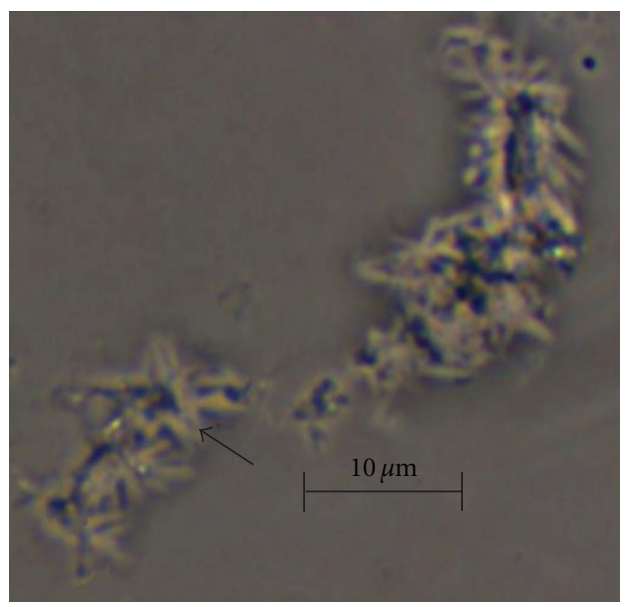

(b)

Figure 3: Micrographs of B. cereus 183 cells treated with Kurstacin 287. Samples were treated with bacteriocin, stained with amido black 10B (BioRad) for 5 min and observed under light microscopy. (a) B. cereus 183 cells without bacteriocin (control), (b) B. cereus 183 cells with Kurstacin 287. Black arrow in (a) and (b) show cells of $B$. cereus not damaged or lysed, respectively.

five bacteriocins of $B$. thuringiensis are not toxic to L. monocytogenes [10], one of the most virulent foodborne pathogen that is the causative agent of the listeriosis. Here, using the fluorogenic method, we demonstrated susceptibility of this bacterium to all bacteriocins tested (Table 2). Likewise, $P$. vulgaris and Shigella flexneri, etiological agents of urinary tract infections and diarrhea in humans, respectively, showed no susceptibility to at least two of the bacteriocins of $B$. thuringiensis as determined by the well-diffusion assay [25], but the antibacterial effect of the bacteriocins was observed with the fluorogenic method (Table 2). Interestingly, we detected antibacterial activity with both methods against Streptococcus agalactiae, one of the most important etiologic agents of mastitis in cattle [30]. To our knowledge, this is the first report on the effect of bacteriocins synthesized by $B$. thuringiensis against this bacterium. We previously report the effect of these bacteriocins against Staphylococcus aureus, a 


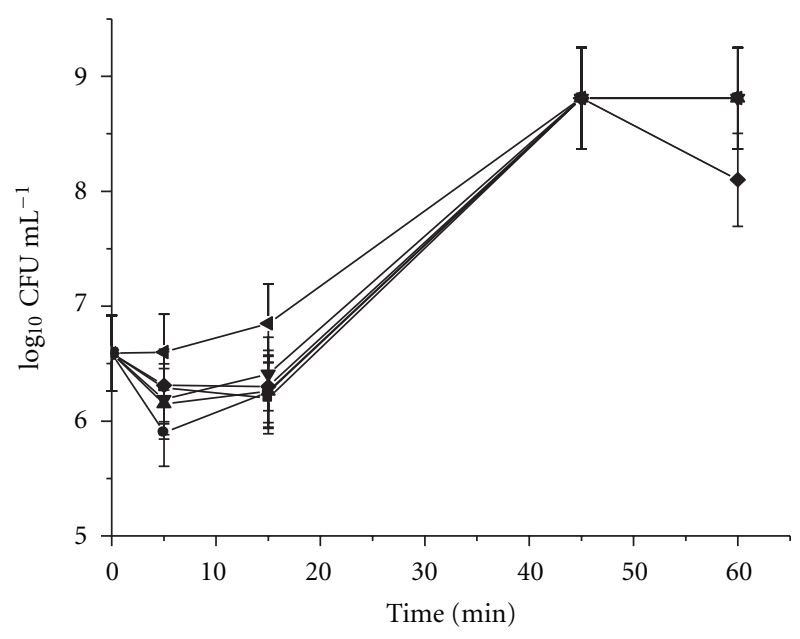

(a)

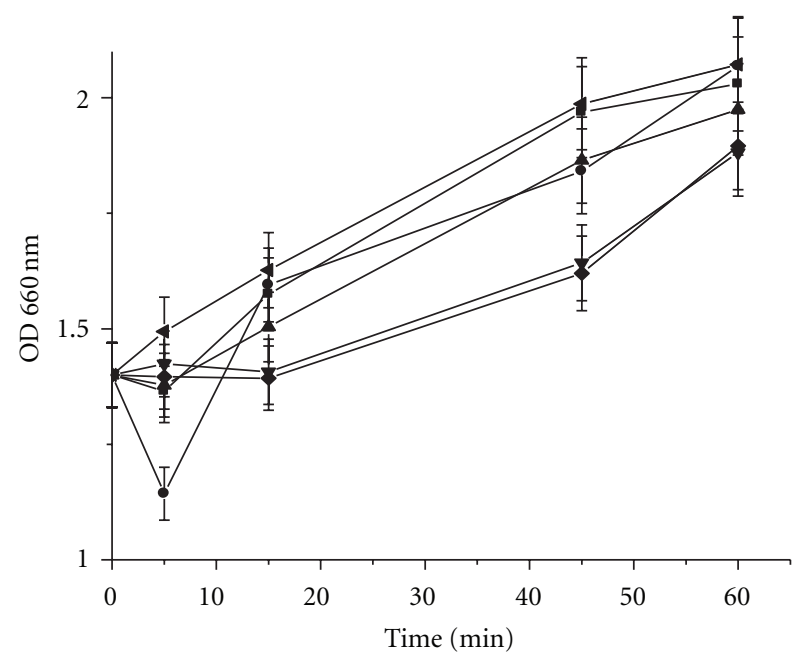

(b)

FIGURE 4: Effect of bacteriocins produced by B. thuringiensis on the growth of Salmonella $s p$. (a) $\log _{10} \mathrm{cfu} \mathrm{mL}^{-1}$ (b) optical density measured at $660 \mathrm{~nm}$. The five bacteriocins were added in the middle of the logarithmic phase of growth curve $(\sim 3 \mathrm{~h})$ of Salmonella sp. culture (indicator strain) used as control (without bacteriocins). Salmonella sp. (४); Morricin 269 (ם); Kurstacin $287(\bullet)$; Kenyacin 404 ( $\mathbf{\Delta})$, Entomocin $420(\boldsymbol{\nabla})$; Tolworthcin $524(\diamond)$.

common human pathogen that is also associated with bovine mastitis [31]. These results suggest the potential applied use of these bacteriocins to control these two etiological agents of mastitis in animals.

The influx of berberine into bacteriocin-treated cells causes the molecule to fluoresce (Figure 1) upon interaction with intracellular components, including DNA and glycosaminoglycans $[17,32,33]$. The mechanisms of action of the bacteriocins produced by $B$. thuringiensis are not known. In particular, data on their interaction with structural components of lipopolysaccharide and peptidoglycan layers are lacking. Although we do not know the amino acid sequence of bacteriocins of this study, it is probably that these antimicrobial peptides have positive charges that could interact with the negative charge of the anionic phospholipids, or with the phosphate groups of lipopolysaccharide located on the outer membrane layer of Gram-negative, and also with the teichoic acids of Grampositive bacteria by electrostatic forces. Such interactions are known to subsequently change the permeability of cellular membrane by a pore formation [34-37]. The pore formation is widespread between cationic bacteriocins such as Thuricin S of B. thuringiensis subsp. entomocidus HD198 [38]. Additionally, it has been observed that bacteriocins can produce different pore sizes in cellular membranes that could be an important factor on the mode of action of the antimicrobial peptide [39]. Regardless, data obtained in this work indicate that bacteriocins of $B$. thuringiensis have both bacteriolytic and bacteriostatic effects, respectively, against Gram-positive and Gram-negative bacteria. It is evident that the absence (i.e., Gram-positive bacteria) or presence (i.e., Gram-negative bacteria) of an outer membrane layer is crucial to define the mode of action (Figures 2 and 3). A dual effect has also been reported with Thuricin 17, Entomocin 110 and Thuricin 7 that have bactericidal-bacteriostatic and bactericidal-bacteriolytic action [7, 11, 18]. Therefore, the presence of an outer lipopolysaccharide membrane of Gram-negative bacteria could be a significant factor that limits efficient intracellular localization of the $B$. thuringiensis bacteriocins studied here.

In conclusion, we have demonstrated that the fluorogenic method is a rapid protocol to determine the antibacterial activity of bacteriocins of $B$. thuringiensis against Grampositive and Gram-negative bacteria, and that the method is a reliable substitute for the traditional well-diffusion method. Moreover, the method could be easily adapted in large-scale screenings of microorganism that produce bacteriocins, and perhaps other antimicrobial agents that disrupt cell wall structures. Our future efforts will focus on purification of bacteriocins of $B$. thuringiensis used in this work, cloning their corresponding genes, and designing methods to mass produce these peptides for both applied and basic biochemical studies, including the mechanism of action.

\section{Authors' Contributions}

Norma M. de la Fuente-Salcido and J. Eleazar Barboza Corona contributed equally to this work.

\section{Acknowledgments}

This research was supported by a grant from the Universidad de Guanajuato (24/11) to J. Barboza-Corona The authors are thankful to Leticia Gabriela Alfaro Martínez, Cristobal Castañeda Ramírez (Universidad de Guanajuato), and Daniel López de la Cruz (Universidad Autónoma de Coahuila) for their technical assistance.

\section{References}

[1] E. Guaní-Guerra, T. Santos-Mendoza, S. O. Lugo-Reyes, and L. M. Terán, "Antimicrobial peptides: general overview and 
clinical implications in human health and disease," Clinical Immunology, vol. 135, no. 1, pp. 1-11, 2010.

[2] J. Delves-Broughton, "Nisin as a food preservative," Food Australia, vol. 57, no. 12, pp. 525-527, 2005.

[3] F. Kamoun, I. B. Fguira, N. B. B. Hassen, H. Mejdoub, D. Lereclus, and S. Jaoua, "Purification and characterization of a new Bacillus thuringiensis bacteriocin active against Listeria monocytogenes, Bacillus cereus and Agrobacterium tumefaciens," Applied Biochemistry and Biotechnology, vol. 165, no. 1, pp. 300-314, 2011.

[4] S. Chehimi, F. Delalande, S. Sablé et al., "Purification and partial amino acid sequence of thuricin $S$, a new anti-Listeria bacteriocin from Bacillus thuringiensis," Canadian Journal of Microbiology, vol. 53, no. 2, pp. 284-290, 2007.

[5] H. Lee, J. J. Churey, and R. W. Worobo, "Biosynthesis and transcriptional analysis of thurincin $\mathrm{H}$, a tandem repeated bacteriocin genetic locus, produced by Bacillus thuringiensis SF361," FEMS Microbiology Letters, vol. 299, no. 2, pp. 205$213,2009$.

[6] A. Cherif, S. Chehimi, F. Limem et al., "Detection and characterization of the novel bacteriocin entomocin 9, and safety evaluation of its producer, Bacillus thuringiensis ssp. Entomocidus HD9," Journal of Applied Microbiology, vol. 95, no. 5, pp. 990-1000, 2003.

[7] A. Cherif, H. Ouzari, D. Daffonchio et al., "Thuricin 7: a novel bacteriocin produced by Bacillus thuringiensis BMG1.7, a new strain isolated from soil," Letters in Applied Microbiology, vol. 32, no. 4, pp. 243-247, 2001.

[8] H. D. Paik, S. S. Bae, S. H. Park, and J. G. Pan, "Identification and partial characterization of tochicin, a bacteriocin produced by Bacillus thuringiensis subsp Tochigiensis," Journal of Industrial Microbiology and Biotechnology, vol. 19, no. 4, pp. 294-298, 1997.

[9] M. C. Rea, C. S. Sit, E. Clayton et al., "Thuricin CD, a posttranslationally modified bacteriocin with a narrow spectrum of activity against Clostridium difficile," Proceedings of the National Academy of Sciences of the United States of America, vol. 107, no. 20, pp. 9352-9357, 2010.

[10] J. E. Barboza-Corona, H. Vázquez-Acosta, D. K. Bideshi, and R. Salcedo-Hernández, "Bacteriocin-like inhibitor substances produced by Mexican strains of Bacillus thuringiensis," Archives of Microbiology, vol. 187, no. 2, pp. 117-126, 2007.

[11] A. Cherif, W. Rezgui, N. Raddadi, D. Daffonchio, and A. Boudabous, "Characterization and partial purification of entomocin 110, a newly identified bacteriocin from Bacillus thuringiensis subsp. Entomocidus HD110," Microbiological Research, vol. 163, no. 6, pp. 684-692, 2008.

[12] S. Morgan, R. P. Ross, and C. Hill, "Bacteriolytic activity caused by the presence of a novel lactococcal plasmid encoding lactococcins A, B, and M," Applied and Environmental Microbiology, vol. 61, no. 8, pp. 2995-3001, 1995.

[13] U. Schillinger and F. K. Lücke, "Antibacterial activity of Lactobacillus sake isolated from meat," Applied and Environmental Microbiology, vol. 55, no. 8, pp. 1901-1906, 1989.

[14] A. Delgado, D. Brito, P. Fevereiro, R. Tenreiro, and C. Peres, "Bioactivity quantification of crude bacteriocin solutions," Journal of Microbiological Methods, vol. 62, no. 1, pp. 121-124, 2005.

[15] N. de la Fuente-Salcido, R. Salcedo-Hernández, M. G. AlanísGuzmán, D. K. Bideshi, and J. E. Barboza-Corona, "A new rapid fluorogenic method for measuring bacteriocin activity," Journal of Microbiological Methods, vol. 70, no. 1, pp. 196-199, 2007.
[16] D. S. Moore, Estadística aplicada básica, Antoni Bosch, 2a edition, 2005.

[17] N. M. De la Fuente-Salcido, R. Salcedo-Hernández, C. Castañeda-Ramírez, V. Cortés-Rodríguez, A. N. EspinoMonzón, and J. E. Barboza-Corona, "Estandarizacion del método fluorogénico para detectar la Actividad de bacteriocinas contra Salmonella spp," Revista Salud Pública y Nutrición, vol. 9, pp. 1441-1410, 2010.

[18] E. J. Gray, K. D. Lee, A. M. Souleimanov et al., "A novel bacteriocin, thuricin 17 , produced by plant growth promoting rhizobacteria strain Bacillus thuringiensis NEB17: isolation and classification," Journal of Applied Microbiology, vol. 100, no. 3, pp. 545-554, 2006.

[19] W. W. Navarre and O. Schneewind, "Surface proteins of grampositive bacteria and mechanisms of their targeting to the cell wall envelope," Microbiology and Molecular Biology Reviews, vol. 63, no. 1, pp. 174-229, 1999.

[20] H. L. Alakomi, E. Skyttä, M. Saarela, T. Mattila-Sandholm, K. Latva-Kala, and I. M. Helander, "Lactic acid permeabilizes gram-negative bacteria by disrupting the outer membrane," Applied and Environmental Microbiology, vol. 66, no. 5, pp. 2001-2005, 2000.

[21] T. Abee, "Bacteriocins: modes of action and potentials in food preservation and control of food poisoning," International Journal of Food Microbiology, vol. 28, no. 2, pp. 169-185, 1995.

[22] L. H. Deegan, P. D. Cotter, C. Hill, and P. Ross, "Bacteriocins: biological tools for bio-preservation and shelf-life extension," International Dairy Journal, vol. 16, no. 9, pp. 1058-1071, 2006.

[23] B. Ray and M. Daeschel, "Food biopreservatives of microbial origin," in Bacteriocins of Starter Culture Bacteria as Food Biopreservatives : An Overview, pp. 177-205, CRC Press, Boca Raton, Fla, USA, 1992.

[24] A. Hurst, "Nisin," Advances in Applied Microbiology, vol. 27, pp. 85-123, 1981.

[25] N. De La Fuente-Salcido, M. Guadalupe Alanís-Guzmán, D. K. Bideshi, R. Salcedo-Hernández, M. Bautista-Justo, and J. E. Barboza-Corona, "Enhanced synthesis and antimicrobial activities of bacteriocins produced by Mexican strains of Bacillus thuringiensis," Archives of Microbiology, vol. 190, no. 6, pp. 633-640, 2008.

[26] C. Castañeda-Ramírez, V. Cortes-rodríguez, N. D. L. FuenteSalcido, D. K. Bideshi, M. C. D. RincóN-Castro, and J. E. Barboza-Corona, "Isolation of Salmonella spp. from lettuce and evaluation of its susceptibility to novel bacteriocins of Bacillus thuringiensis and antibiotics," Journal of Food Protection, vol. 74, no. 2, pp. 274-278, 2011.

[27] T. J. Montville, H. J. Chung, M. L. Chikindas, and Y. Chen, "Nisin A depletes intracellular ATP and acts in bactericidal manner against Mycobacterium smegmatis," Letters in Applied Microbiology, vol. 28, no. 3, pp. 189-193, 1999.

[28] K. A. Stevens, B. W. Sheldon, N. A. Klapes, and T. R. Klaenhammer, "Nisin treatment for inactivation of Salmonella species and other gram- negative bacteria," Applied and Environmental Microbiology, vol. 57, no. 12, pp. 3613-3615, 1991.

[29] T. J. Montville and M. E. C. Bruno, "Evidence that dissipation of proton motive force is a common mechanism of action for bacteriocins and other antimicrobial proteins," International Journal of Food Microbiology, vol. 24, no. 1-2, pp. 53-74, 1994.

[30] P. L. Ruegg, "Investigation of mastitis problems on farms," Veterinary Clinics of North America, vol. 19, no. 1, pp. 47-73, 2003.

[31] J. E. Barboza-Corona, N. de la Fuente-Salcido, N. AlvaMurillo, A. Ochoa-Zarzosa, and J. E. López-Meza, "Activity 
of bacteriocins synthesized by Bacillus thuringiensis against Staphylococcus aureus isolates associated to bovine mastitis," Veterinary Microbiology, vol. 138, no. 1-2, pp. 179-183, 2009.

[32] R. Reyes, G. Ramírez, and N. M. Delgado, "Fluorescent berberine binding as a marker of internal glycosaminoglycans sulfate in bovine oocytes and sperm cells," Archives of Andrology, vol. 50, no. 5, pp. 327-332, 2004.

[33] S. S. Talwalkar, A. B. Vaidya, C. Godse, A. Vaidya, and R. Vaidya, "Plasmodium DNA fluoresces with berberine: a novel approach for diagnosis of malarial parasites," American Journal of Clinical Pathology, vol. 124, no. 3, pp. 408-412, 2005.

[34] J. P. Bradshaw, "Cationic antimicrobial peptides: issues for potential clinical use," BioDrugs, vol. 17, no. 4, pp. 233-240, 2003.

[35] H. G. Boman, "Peptide antibiotics and their role in innate immunity," Annual Review of Immunology, vol. 13, pp. 61-92, 1995.

[36] H. G. Boman, "Antibacterial peptides: basic facts and emerging concepts," Journal of Internal Medicine, vol. 254, no. 3, pp. 197-215, 2003.

[37] R. E. W. Hancock and A. Rozek, "Role of membranes in the activities of antimicrobial cationic peptides," FEMS Microbiology Letters, vol. 206, no. 2, pp. 143-149, 2002.

[38] S. Chehimi, A. M. Pons, S. Sablé, M. R. Hajlaoui, and F. Limam, "Mode of action of thuricin S, a new class IId bacteriocin from Bacillus thuringiensis," Canadian Journal of Microbiology, vol. 56, no. 2, pp. 162-167, 2010.

[39] Y. Gao, M. J. Van Belkum, and M. E. Stiles, "The outer membrane of gram-negative bacteria inhibits antibacterial activity of brochocin-C," Applied and Environmental Microbiology, vol. 65, no. 10, pp. 4329-4333, 1999. 

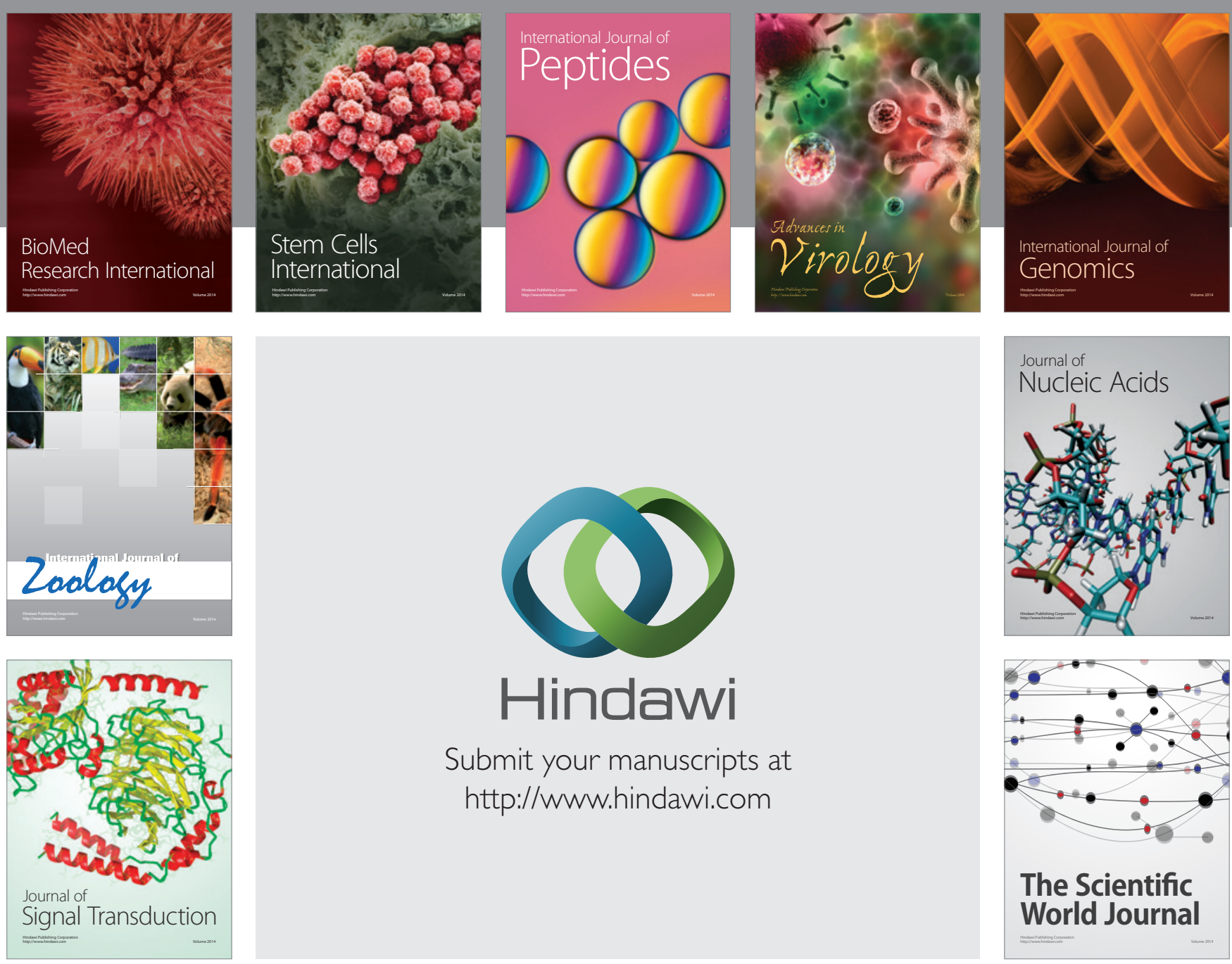

Submit your manuscripts at

http://www.hindawi.com
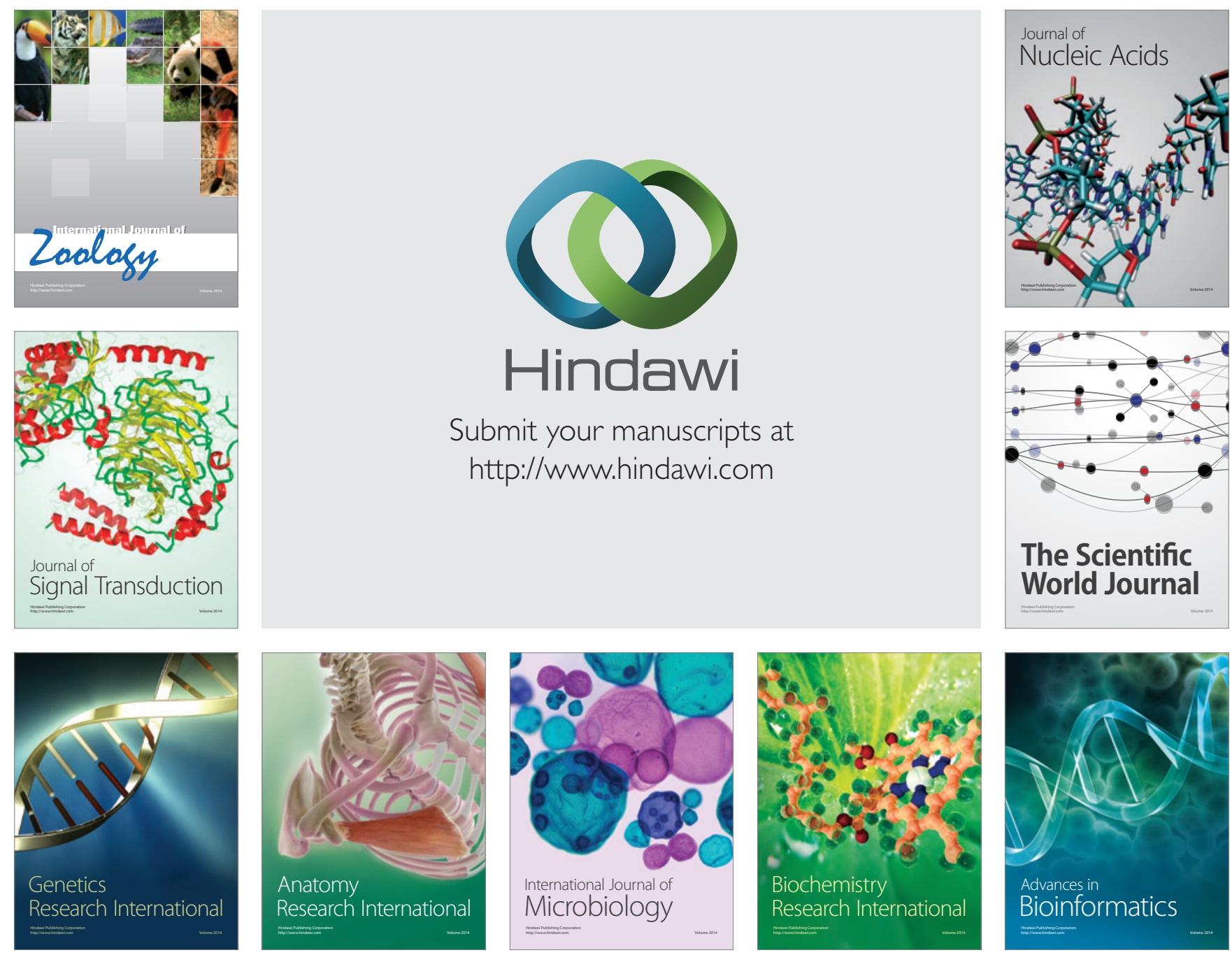

The Scientific World Journal
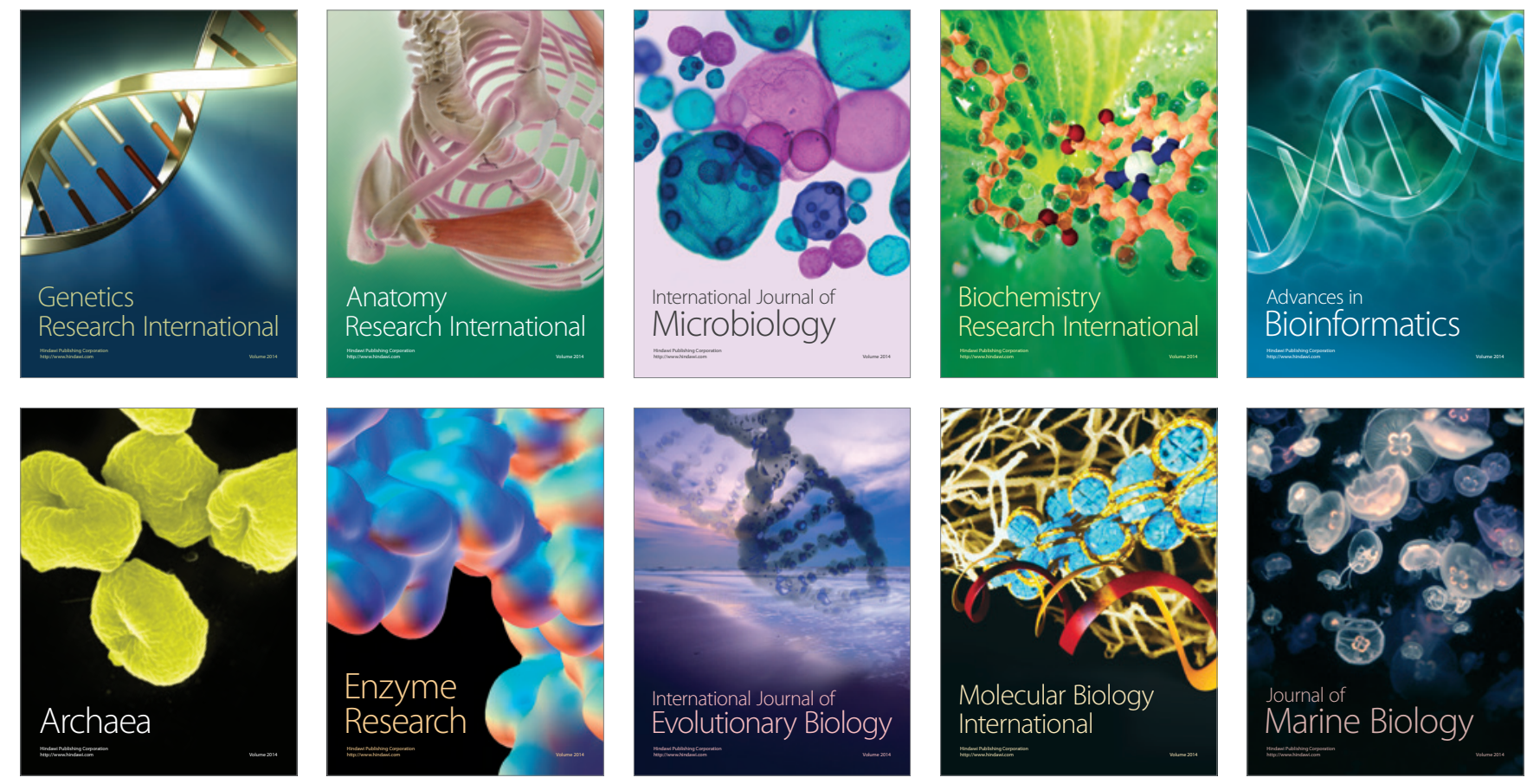